\title{
Snakes of the United States and Canada: Natural History and Care in Captivity
}

By John Rossi and Roxanne Rossi. 2003. Krieger Publishing Company, Florida. xxxvi + 520 pages. U.S. \$145.

This substantial hardcover builds on the Rossis' previous two-volume set on the captive care of snakes, published in 1995. The authors have revised some information based on new data and have also expanded the scope of the book to include basic information on the natural history of each species, resulting in a single authoritative volume covering all currently recognised species within the continental United States and Canada. The price of this book, combined with its ambitious scope, may deter novice or casual herpetoculturalists and herpetologists from adding it to their collections; however, its well-written, accessible style and wealth of "hands-on" experience make it a valuable resource for anyone with an interest in our native North American snakes.

Dr. John Rossi is a veterinarian specialising in the care of small animals and "exotics," with a particular interest in herpetofauna. His wife Roxanne is an experienced curator, whose first-hand knowledge is evident throughout the discussions on captive care. Together, the Rossis have kept over 90 of the native species discussed in the book, and have published numerous articles and books based on their observations. They have also undertaken extensive research and consultation with other experienced keepers, as evidenced by the impressive list of acknowledgments and the 21-page bibliography. The result is a thorough reference document, which nonetheless recognises (and promotes) the need for additional investigation into certain less well-known species.

The Rossis' passion for their topic is evident throughout the volume, beginning with the introduction and overview of the natural history of snakes. Their position on captive care as a source of valuable information and understanding, which could assist conservation efforts, is a recurring theme within the book. A short chapter on conservation is included in the introductory section of the book, which touches on the issues of habitat loss and fragmentation, road kills, pollution and human attitudes towards snakes (fear and loathing vs. overzealous collecting by hobbyists and traders).

Part 1 of the main body of the text deals with general care information for the keeping of snakes. Housing is the first subject dealt with, based on the reasoning that prior to obtaining any snake, the owner should first have a suitable home ready for it. Various types of housing set-ups are described in detail, along with colour photographs and a summary table of suitable enclosure sizes (based on standard aquarium sizes). This section also covers the other basic components of captive care, from selecting, obtaining and transporting the chosen snake, to keeping, handling, feeding and breeding it. The discussion on reproduction includes methods of sexing the snake, tips for successful breed- ing, and instructions on rearing neonates. A summary table presents information on breeding season, clutch size and incubation period for the various species. The Rossis emphasize the importance of keeping accurate records, both to the individual keeper and to herpetology as a whole when these records are published. They also discuss the circumstances under which a captive snake should or should not be released back into the wild.

The individual species accounts are presented in two parts. Part 2 describes the nonvenomous snakes (including inoffensive rear-fanged species such as hognose snakes, night snakes, etc.) while Part 3 deals with the venomous snakes. These sections are arranged alphabetically by scientific name; for ease of reference, the book also includes an annotated list of genera preceding Part 1, and an Appendix listing accepted common names and their associated scientific binomial. Part 3 is prefaced with a brief cautionary introduction to the essential differences in technique required when keeping, transporting and maintaining venomous species. Each species account provides a brief summary of reported information on distribution, habitat, physical characteristics, prey and predators, and reproduction in the wild, accompanied by a range map (primarily continental United States/southern Canada, adjusted as necessary for wide-ranging species like Common Garter). This discussion on natural history is followed by captive care information including tips from the Rossis' experience on preferred housing, food, and other requirements. Notes on potential feeding or health problems are also included. Superb colour photographs enhance each species account.

A summary of the species' basic needs and Maintenance Difficulty Index (MDI) is also included in each species account for quick reference and comparison between species. The MDI indicates a species' relative difficulty level, based on the authors' experience. A rating of 1 is applied to species suitable for novices, such as the hugely popular Corn Snake. More challenging species may have highly specific habitat requirements or hard-to-obtain food preferences. The most difficult species are rated as having an MDI of 5 . The explanation of the MDI is somewhat difficult to find within the discussion on choosing a species in Part 1 (a sample species information summary, with explanations of the types of data presented therein, might have made a good addition to the book). However, this minor difficulty is offset by the inclusion of a reminder of the ranking system $(1=$ easiest, $5=$ most difficult) in each species' information summary. Other information included in the summary is the species name, average adult length, appropriate food items, cage size, substrate, heat and light sources, temperature range (in Celsius and Fahrenheit), and special considerations such as any potential health issues to watch for. 
In conclusion, Snakes of the United States and Can$a d a$ is an impressive compilation of natural history and captive care data for our native North American species. For those readers interested in supplementing their collection of field guides or species care handbooks, this book is a worthwhile acquisition.

\section{Stonechats: A Guide to the Genus Saxicola}

By E. Urquhart and A. Bowley. 2002. Yale University Press, New Haven and London. 320 pages. U.S. \$60. Cloth.

This book is delightful and does the Saxicola genus a great service. It covers 14 members of this genus from the Old World, which is as fascinating as it can get for birders since it deals with countries like India, Nepal, Russia, Indonesia and with various other fascinating locations in Europe and Africa.

Obviously, this book is a great reflection of the so entrenched English and European birding culture, and the author links also well to Russian, German and Scandinavian Saxicola studies. The explicit goal of this book is to provide as much information on the Stonechat species as possible. The authors achieved in their book a huge compilation of a tremendous amount of information and references relevant to Stonechats such as Nest Record Cards from the British Trust for Ornithology (BTO), BTO Migration Atlas, World Bird Database by BirdLife International, 10 museum collections and many others; for instance, over 173 individual investigators are mentioned in the acknowledgment section of this publication. This makes for a text crowded with details.

The very detailed accounts for the 14 species and their subspecies deal with taxonomy, identification, description, distribution, breeding, habitat, voice, food, movements, behaviour, moult, conservation and literature references.

From a biologist's perspective, some of the presented distribution maps might have too many straight lines, but they show great detail in regards to extralimital sightings, wintering, summer and subspecies distributional ranges (but not search effort and migration flyways). The limitations of these maps are also well addressed and explained. For people with an interest in avian biogeography, this book presents a large goldmine consisting of fascinating endemism and distribution data, e.g. for White-tailed Stonechat and Pied Bushchat. The text and presented data offer great opportunities for further spatial interpretation and predictive modeling. A comprehensive World Distribution Map for the genus Saxicola is unfortunately missing.

Tables showing wing, tail and bill measurements are also presented but some do not provide sample sizes. The voice sections and sonograms are nice summaries on what is known on the vocalization for each species. Often, the presented vocalization data cast serious doubt on the traditional concept of point count surveys and habitat mapping for these species since they
Furthermore, anyone considering snake ownership would be well advised to at least borrow a copy.

Amy MacPherson

2781 Lancaster Road, Suite 200, Ottawa, Ontario K1B 1A7 Canada

can sing during migration even, and also mimic other species brilliantly; often also both genders can sing! The authors' concept that only birders would decide which exact bird names are used eventually for bird species world-wide might still be open for discussion. Anyway, it's great to read the section provided on explanations of English and scientific names.

Remarkable are the extremely powerful and very well done colour plates and the many smaller fascinating black-and-white sketches displaying specific behaviours. They are based on great field observations by A. Bowley and E. Urquhart. Over 100 photographs are provided as well allowing for excellent visual aids in species identification.

For my taste, some of the interpretations of the reported research findings are too nationalistic, e.g. stating that birds in Germany, in the former USSR, in Greece, in UK etc. would do specific things. Besides presenting a generalization, such an interpretation does not consider that the area size differs so much among countries. Instead, it might be better to state findings from a sample of a population or from studied individuals.

The authors say that "....many younger birders now only concentrate on identifying and seeing as many different species as possible, without taking time to study the all-round ecology of the species they see". The book includes a short but informative Saxicola DNA chapter by Wink et al. dealing with long lists of TCAs, RAAs, CGAs etc. which I cannot fully comprehend; hopefully the birder in the field can. The authors suggest to split the Common Stonechat complex into three species. Generally, one finds birders and geneticists closely linked nowadays, but for me they represent odd bed-fellows. Geneticists dictate a lot of what happens in the birding field nowadays. However, so far avian geneticists have not always clarified situations but rather made amateur birders more dizzy in what constitutes a species, a subspecies and who is evolutionarily related to whom.

This book includes 11 pages of international Stonechat literature references. This provides for a text which offers much fascinating reading such as for instance for the Stolizka's Bushchat and for the highly endangered Hodgson's Bushchat. Interestingly enough, albinism occurs in the genus Saxicola whereas leucism appears to be non-existent in the literature and is probably very rare. Presented conservation topics in this book deal for instance with the stability or decline of national Saxicola populations, but caution is to be 